\title{
On the Hughes Model and Numerical Aspects
}

\author{
Diogo A. Gomes and Roberto M. Velho
}

\begin{abstract}
We study a crowd model proposed by R. Hughes in [11] and we describe a numerical approach to solve it. This model comprises a Fokker-Planck equation coupled with an eikonal equation with Dirichlet or Neumann data. First, we establish a priori estimates for the solutions. Second, we study radial solutions and identify a shock formation mechanism. Third, we illustrate the existence of congestion, the breakdown of the model, and the trend to the equilibrium. Finally, we propose a new numerical method and consider two examples.
\end{abstract}

\section{INTRODUCTION}

Understanding the dynamics of pedestrian crowds is of great significance for the prevention of catastrophic emergency evacuations. Here, we consider an extension of the partial differential equation (PDE) model in [11] that describes the evolution of a pedestrian crowd. Our system of PDEs comprises a continuity equation or Fokker-Planck equation with viscosity $\varepsilon \geq 0$ coupled to an Eikonal equation

$$
\left\{\begin{array}{l}
\rho_{t}(x, t)-\operatorname{div}\left(\rho(1-\rho)^{2} D u\right)=\varepsilon \Delta \rho, \\
|D u(x)|^{2}=\frac{1}{(1-\rho)^{2}},
\end{array}\right.
$$

where $\rho: \Omega \times \mathbb{R}_{+} \rightarrow \mathbb{R}$, the density of agents, and $u: \Omega \rightarrow \mathbb{R}$, the time to exit $\Omega$ through its boundary $\partial \Omega$, are the unknowns and the given initial data is $\rho(x, 0)=\rho_{0}(x)$ and $u(x)=u_{0}$, with $x$ representing the spatial variable and $t$ the time. Also $\rho_{t}$ stands for the time derivative of $\rho$; $D u$ and $\Delta u$ the spatial gradient and the Laplacian of $u$ and $u_{\nu}$, $\rho_{\nu}$ are the directional derivatives of $u$ and $\rho$ with respect to the outer unit normal vector to $\partial \Omega$. We also suppose $\Omega$ is an open domain of $\mathbb{R}^{d}$, with $\partial \Omega=\Gamma_{d} \cup \Gamma_{n}$, the Dirichlet and the Neumann parts of the boundary respectively which are the relevant boundary conditions in applications and $d \leq 3$. The Fokker-Planck equation describes the evolution of the crowd density $\rho(0 \leq \rho \leq 1)$. The solution to Eikonal equation represents the time an agent/person/pedestrian takes to exit the domain $\Omega$ taking into account congestion effects; its gradient, $D u$, determines the optimal direction of movement for each agent (in a steepest descent way) assuming the rest of the population is frozen (due to the stationary character of the Eikonal). The constraint $\rho \leq 1$ corresponds to the maximal density of the population. The Dirichlet condition ( $\rho$ or $u$ prescribed on $\partial \Omega$ ) corresponds to the areas on the

This work was partially supported by KAUST baseline and start-up funds and KAUST SRI, Uncertainty Quantification Center in Computational Science and Engineering.

King Abdullah University of Science and Technology (KAUST), CEMSE Division and KAUST SRI, Uncertainty Quantification Center in Computational Science and Engineering, Thuwal 23955-6900, Saudi Arabia. diogo.gomesakaust.edu.sa roberto.velho@gmail.com boundary where agents/people/pedestrians can leave. The Neumann condition $\left(\rho_{\nu}\right.$ and $u_{\nu}$ prescribed on $\partial \Omega$ ) is a noflow constraint at the boundary. The correct interpretation of these boundary conditions is essential in the design of numerical schemes. The case $\varepsilon=0$ is the model in [11].

Significant progress has been achieved in the understanding of these problems ([1], [7], [2], [8]). However, even some aspects of one-dimensional models are not completely understood. Microscopic ([5], [6]) and mean-field game ([3], [12]) interpretations were used to study the macroscopic dynamics that the Hughes model describes. Numerical approaches to these problems were developed in [4], [9].

In section II, we establish new a priori estimates for solutions of (1) that give a partial regularity for the solutions. Then, in section III, we consider a radial problem to examine the behavior of the model in two and three dimensions in under a simplified setting. We show that with zero viscosity $(\varepsilon=0)$, the model admits shocks, which we also illustrate numerically. The shocks represent situations where nearby agents follow a substantially different direction or where there are discontinuities in the density $\rho$. The presence of shocks makes the study of numerical methods and of the existence of solutions into non-trivial problems. Next, we study a one-dimensional problem, the flow problem, in section IV. Here, agents arrive at a prescribed rate, that we call current, on one side of an interval and leave through the other side. We show that the Hughes model may not be wellposed as the density $\rho$ can exceed the maximal congestion threshold. In these examples, we identify two mechanisms of loss of regularity: shocks in the zero-viscosity problem and congestion in the flow problem. Finally, we illustrate the trend to equilibrium in section $\mathrm{V}$ and, in section VI, we discuss a new numerical method for the solution of (1) and two numerical examples. As a last remark, we point out that, in the general Hughes model, the r.h.s. of the Eikonal equation is of the form $1 / f(\rho)$ for a generic $f$. Here, we do not study this problem but the core difficulties are similar. Thus, we believe that both negative results (existence of shocks, model breakdown) and positive ones (estimates, numerical scheme) can be treated with a similar approach.

\section{ESTIMATES}

We begin our study of the Hughes model by investigating a priori estimates; that is, estimates that are valid for smooth enough solutions. These estimates extend the ones in [10] for the periodic setting. First, for the Neumann or Dirichlet boundary conditions and $\varepsilon>0$, we prove that smooth solutions satisfy $0 \leq \rho \leq 1$ for all times if the initial condition also satisfies this condition. This should 
be contrasted with the flow problem considered in Section IV. Next, we prove that $D u \in L^{p}$ (Lebesgue space) for any $1<p<\infty$, and then $\frac{1}{1-\rho} \in L^{p}$. This last estimate gives a quantitative control on the congestion. Here, in contrast, we consider the Hughes model in an open domain, $\Omega \subset \mathbb{R}^{d}$, with Dirichlet-Neumann conditions: $\partial \Omega=\Gamma_{d} \cup \Gamma_{n}, \nu$ the outer unit normal to $\partial \Omega, \rho=0$ in $\Gamma_{d} \times[0, T], \rho_{\nu}=0$ in $\Gamma_{n} \times[0, T]$ and viscosity $\varepsilon=1$.

Lemma 1: Let $\rho: \Omega \times[0, T] \rightarrow \mathbb{R}$ solve

$$
\rho_{t}-\operatorname{div}(\rho(1-\rho) g(x, t))=\Delta \rho
$$

where $\Omega$ is an open subset of $\mathbb{R}^{d}$ with $\partial \Omega=\Gamma_{d} \cup \Gamma_{n}, \rho=0$ in $\Gamma_{d} \times[0, T]$, and $\rho_{\nu}=0$ in $\Gamma_{n} \times[0, T]$. Assume also $0 \leq \rho(x, 0) \leq 1$ and $g \in C^{\infty}$. Then $0 \leq \rho(x, t) \leq 1$.

Proof: Note that $\tilde{\rho}=1-\rho$ satisfies

$$
\tilde{\rho}_{t}-\operatorname{div}(\rho \tilde{\rho} g(x, t))=\Delta \tilde{\rho} .
$$

Because $\tilde{\rho}(x, 0) \geq 0$ (and, with Dirichlet boundary data, $\tilde{\rho} \geq 0$ in $\partial \Omega$ ), we have $\tilde{\rho} \geq 0$.

Proposition 1: Let $(u, \rho)$ solve (1) with $\varepsilon=1$. Suppose $u=0$ in $\Gamma_{d} \times[0, T], u_{\nu}=0$ on $\Gamma_{n} \times[0, T]$ and $0<\rho<1$ at $t=0$. Then, for any $\alpha<-1$

$$
\frac{d}{d t} \int_{\Omega}(1-\rho)^{\alpha+1} \leq C \int_{\Omega}(1-\rho)^{\alpha+1}
$$

Furthermore,

$$
\int_{0}^{T} \int_{\Omega}\left|D(1-\rho)^{\frac{\alpha+1}{2}}\right|^{2} \leq C .
$$

Proof: Multiply the first equation in (1) by $-(\alpha+1)(1-\rho)^{\alpha}$. Then,

$$
\begin{aligned}
& \frac{d}{d t} \int_{\Omega}(1-\rho)^{\alpha+1} \leq c \int_{\Omega}(1-\rho)^{\alpha+1} \rho D \rho D u \\
& -\alpha(\alpha+1) \int_{\Omega}(1-\rho)^{\alpha-1}|D \rho|^{2} \\
& -(\alpha+1) \int_{\partial \Omega}(1-\rho)^{\alpha+2} \rho u_{\nu}-(\alpha+1) \int_{\partial \Omega}(1-\rho)^{\alpha} \rho_{\nu}
\end{aligned}
$$

(using Cauchy's inequality)

$$
\begin{aligned}
& \leq-\frac{\alpha(\alpha+1)}{2} \int_{\Omega}(1-\rho)^{\alpha-1}|D \rho|^{2}+\int_{\Omega}(1-\rho)^{\alpha+3} \rho^{2}|D u|^{2} \\
& -(\alpha+1) \int_{\partial \Omega}(1-\rho)^{\alpha+2} \rho u_{\nu}-(\alpha+1) \int_{\partial \Omega}(1-\rho)^{\alpha} \rho_{\nu}
\end{aligned}
$$

(using the Eikonal eq. and the Lemma 1)

$$
\begin{aligned}
& \leq-\frac{\alpha(\alpha+1)}{2} \int_{\Omega}(1-\rho)^{\alpha-1}|D \rho|^{2}+C \int_{\Omega}(1-\rho)^{\alpha+1} \\
& -(\alpha+1) \int_{\partial \Omega}(1-\rho)^{\alpha+2} \rho u_{\nu}-(\alpha+1) \int_{\partial \Omega}(1-\rho)^{\alpha} \rho_{\nu} .
\end{aligned}
$$

Now, we observe that, on $\Gamma_{n}, u_{\nu}=0$ and, on $\Gamma_{d}$, we have $\rho=0$. Hence, $(1-\rho)^{\alpha+2} \rho u_{\nu}=0$ in $\partial \Omega$. Similarly, $(1-\rho)^{\alpha} \rho_{\nu} \leq 0$ in $\Gamma_{d}$ and vanishes in $\Gamma_{n}$. Hence, is also non-positive in $\partial \Omega$. Thus, taking into account that $\alpha+1 \leq 0$, integrating in time, observing that the only positive term on the r.h.s. is $\int(1-\rho)^{\alpha+1}$, and using Gronwall's inequality, we get the desired estimates.

Proposition 2: Under the same hypothesis of Proposition 1 , we have that, for any $1<p<\infty$,

$$
\sup _{0 \leq t \leq T} \int_{\Omega}|D u|^{2 p}<C_{p}
$$

Proof: By using (2) in the Eikonal equation, we have

$$
|D u|^{2}=\frac{1}{(1-\rho)^{2}} \in L^{p}, \forall p .
$$

\section{SHOCKS IN RADIAL SOLUTIONS}

To understand the behavior of the Hughes model, we consider radial solutions. Thus (1) becomes a scalar PDE. Thanks to this simplification, we identify the formation of shocks in the zero viscosity problem. We expect shocks to exist in general 2 or 3-dimensional problems because radial solutions are a particular case.

Now, we assume radial symmetry corresponding to a model where agents want to get away from the origin. In dimension $d>1$, assume $u=u(r, t)$, where $r$ is the radius. The eikonal equation in (1) gives that

$$
u_{r}= \pm \frac{1}{1-\rho} \text {. }
$$

We select the negative root because it corresponds to agents leaving the origin. Because $\rho$ is radial, $\rho=\rho(r, t)$, we rewrite the Fokker-Planck equation in (1) in polar coordinates and using the preceding equation, we get

$$
\rho_{t}+\frac{d-1}{r} \rho(1-\rho)+\rho_{r}(1-2 \rho)=\varepsilon\left[\rho_{r r}+\frac{d-1}{r} \rho_{r}\right] \text {. }
$$

When $\varepsilon=0$, we obtain the first-order PDE

$$
\rho_{t}+\frac{d-1}{r} \rho(1-\rho)+\rho_{r}(1-2 \rho)=0 .
$$

As it is usual for first-order nonlinear PDEs, shocks can arise. We study the shocks using the method of characteristics.

First, we solve the characteristic system

$$
\left\{\begin{array}{l}
\frac{d r}{d t}=1-2 \rho \\
\frac{d \rho}{d t}=-\frac{d-1}{r} \rho(1-\rho)
\end{array}\right.
$$

with $r_{0}, \rho_{0}\left(r_{0}\right)$ as initial conditions at $t=0$ for $r(t)$ and $\rho(t)$, respectively.

To solve this system of ODEs, we first define $V$ as

$$
V(\rho)=\rho(1-\rho)
$$

and, analogously, $V_{0}\left(\rho_{0}\right)=\rho_{0}\left(1-\rho_{0}\right)$. Next, using implicit differentiation, we rewrite (5) as

$$
\left\{\begin{array}{l}
\frac{d r}{d t}=\frac{\partial V}{\partial \rho} \\
\frac{d \rho}{d t}=-\frac{d-1}{r} V
\end{array}\right.
$$


Then, we compute $\frac{\partial V}{\partial r}=-\frac{d-1}{r} V$, and conclude that

$$
V=\left(\frac{r_{0}}{r}\right)^{d-1} V_{0} .
$$

Now, solving for $\rho$ in (6) gives two roots. If $0<\rho_{0}\left(r_{0}\right) \leq \frac{1}{2}$, here called regime 1 ,

$$
\rho_{1}=\frac{1-\sqrt{1-4 V}}{2},
$$

while, if $1 / 2<\rho_{0}\left(r_{0}\right)<1$, here called regime 2 ,

$$
\rho_{2}=\frac{1+\sqrt{1-4 V}}{2} .
$$

Using these expressions on the R.H.S. of the ordinary differential equation (ODE) for $r(t)$ (5) and (8), we get

$$
\dot{r_{1}}(t)=1-2 \rho_{1}=\sqrt{1-4 V_{0}\left[\frac{r_{0}}{r_{1}(t)}\right]^{d-1}},
$$

and

$$
\dot{r_{2}}(t)=1-2 \rho_{2}=-\sqrt{1-4 V_{0}\left[\frac{r_{0}}{r_{2}(t)}\right]^{d-1}} .
$$

Now, using (7) and (8), we obtain the ODE describing the time evolution of $\rho$ :

$$
\dot{\rho}(t)=-\frac{d-1}{r(t)}\left[\frac{r_{0}}{r(t)}\right]^{d-1} V_{0} .
$$

Along the next two subsections, we present the particular cases of the radial solutions in dimension 2 and 3 . We also make use of the following remark.

Remark 1: The function defined by $x \mapsto \sqrt{1-4 x(1-x)}$, is identical to $-2 x+1$ in $[0,1 / 2]$ and identical to $2 x-1$ in $[1 / 2,1]$.

\section{A. Dimension 2}

For the Hughes model in dimension 2, we solve:

$$
\begin{gathered}
\dot{r_{1}}(t)=\sqrt{1-4 V_{0} \frac{r_{0}}{r_{1}(t)}}, \text { with } 0<\rho_{0}\left(r_{0}\right) \leq 1 / 2, \\
\dot{r_{2}}(t)=-\sqrt{1-4 V_{0} \frac{r_{0}}{r_{2}(t)}}, \text { with } 1 / 2<\rho_{0}\left(r_{0}\right)<1,
\end{gathered}
$$

with the initial conditions $r_{1}(0)=r_{2}(0)=r_{0}$. We have the following implicit representation for $r_{1}(t)$ and $r_{2}(t)$ :

Regime 1: $r_{1}(t)$ satisfies

$$
\begin{aligned}
& 2 r_{0} \rho_{0}\left(1-\rho_{0}\right) \log \left[2 r_{1}(t)\left(1+\sqrt{1-\frac{4 r_{0} \rho_{0}\left(1-\rho_{0}\right)}{r_{1}(t)}}\right)\right. \\
& \left.-4 r_{0} \rho_{0}\left(1-\rho_{0}\right)\right]+r_{1}(t) \sqrt{1-\frac{4 r_{0} \rho_{0}\left(1-\rho_{0}\right)}{r_{1}(t)}} \\
& =t+r_{0}\left\{2 \rho_{0}\left(1-\rho_{0}\right) \log \left[4 r_{0}\left(1-\rho_{0}\right)^{2}\right]-2 \rho_{0}+1\right\} .
\end{aligned}
$$

Regime 2: $r_{2}(t)$ satisfies

$$
\begin{aligned}
& 2 r_{0} \rho_{0}\left(1-\rho_{0}\right) \log \left[2 r_{2}(t)\left(1+\sqrt{1-\frac{4 r_{0} \rho_{0}\left(1-\rho_{0}\right)}{r_{2}(t)}}\right)\right. \\
& \left.-4 r_{0} \rho_{0}\left(1-\rho_{0}\right)\right]+r_{2}(t) \sqrt{1-\frac{4 r_{0} \rho_{0}\left(1-\rho_{0}\right)}{r_{2}(t)}} \\
& =-t+r_{0}\left\{2 \rho_{0}\left(1-\rho_{0}\right) \log \left[4 r_{0} \rho_{0}^{2}\right]+2 \rho_{0}-1\right\},
\end{aligned}
$$

In dimension 2 , the equation for the time evolution of $\rho$, equation (11), becomes

$$
\dot{\rho}(t)=-\frac{r_{0}}{r(t)^{2}} V_{0} .
$$

The preceding equation also gives an implicit representation for $\rho$. Here, we solve it numerically and present the parametric plot of the radius $r(t)$ versus the density $\rho(t)$ in subsection III-C.

\section{B. Dimension 3}

For the Hughes model in dimension 3, we solve:

$$
\begin{gathered}
\dot{r_{1}}(t)=\sqrt{1-\frac{4 V_{0} r_{0}^{2}}{r_{1}(t)^{2}}}, \text { with } 0<\rho_{0}\left(r_{0}\right) \leq 1 / 2, \\
\dot{r_{2}}(t)=-\sqrt{1-\frac{4 V_{0} r_{0}^{2}}{r_{2}(t)^{2}}}, \text { with } 1 / 2<\rho_{0}\left(r_{0}\right)<1,
\end{gathered}
$$

with the initial conditions $r_{1}(0)=r_{2}(0)=r_{0}$ and obtain the explicit formulas

$$
\begin{aligned}
& r_{1}(t)=\sqrt{4 r_{0}^{2} V_{0}+t^{2}+2 t C_{1}+C_{1}^{2}}, \\
& r_{2}(t)=\sqrt{4 r_{0}^{2} V_{0}+t^{2}-2 t C_{2}+C_{2}^{2}},
\end{aligned}
$$

where $C_{1}=C_{2}= \pm r_{0} \sqrt{1-4 V_{0}}$ because $r(0)=r_{0}$.

Remark 1 then implies that $C_{1}=r_{0}\left(-2 \rho_{0}+1\right)$ and $C_{2}=r_{0}\left(2 \rho_{0}-1\right)$. Plugging them back in the expressions for $r_{1}(t), r_{2}(t)$ we obtain:

$$
\begin{aligned}
& r_{1}(t)=\sqrt{t^{2}+2 r_{0}\left(1-2 \rho_{0}\right) t+r_{0}^{2}}, \\
& r_{2}(t)=\sqrt{t^{2}-2 r_{0}\left(2 \rho_{0}-1\right) t+r_{0}^{2}} .
\end{aligned}
$$

Because the expressions for $r_{1}(t)$ and $r_{2}(t)$ agree, there is no need to consider two separate regimes. Thus, we get

$$
r(t)=\sqrt{t^{2}+2 r_{0}\left(1-2 \rho_{0}\right) t+r_{0}^{2}} .
$$

Now, the ODE for the density $\rho(t)(11)$ is

$$
\dot{\rho}(t)=-2 \frac{r_{0}{ }^{2}}{r(t)^{3}} V_{0} .
$$

The solution with the initial condition $\rho(0)=\rho_{0}$ is

$$
\begin{aligned}
\rho(t) & =\frac{1}{2}\left[1-\frac{t+r_{0}\left(1-2 \rho_{0}\right)}{r(t)}\right] \\
& =\frac{1}{2}\left[1-\frac{t+r_{0}\left(1-2 \rho_{0}\right)}{\sqrt{t^{2}+2 r_{0}\left(1-2 \rho_{0}\right) t+r_{0}^{2}}}\right] .
\end{aligned}
$$




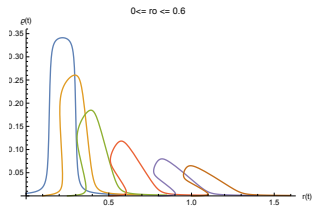

(a) Case 1: $\rho_{0}$ concentrated around 0.2 with support on $0 \leq r_{0} \leq 0.6$ and maximum value of 0.35 .

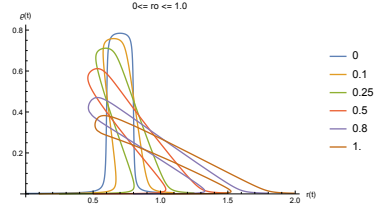

(b) Case 2: $\rho_{0}$ concentrated around 0.75 with support on $0.5 \leq r_{0} \leq 1.0$ and maximum value of 0.8 .

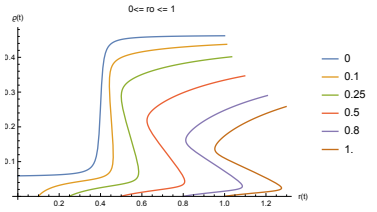

(c) Case 3: $\rho_{0}$ with support on $0 \leq r_{0} \leq 1.0$ and maximum value of 0.4 .

Fig. 1: Shocks along the time evolution of three given profiles in 2-D.

\section{Numerical experiments}

Now, we numerically investigate the formation of shocks for the radial Hughes model without viscosity. We construct three density profiles, $\rho_{0}\left(r_{0}\right)$, with support in $[0,1]$. For each profile, we plot the graphs of $r(t)$ versus $\rho(t)$. The different colors correspond to the solution at the different times. The blue curve in each plot is the initial density profile $\rho_{0}$, see Figure 1 for the two-dimensional case and Figure 2 for the three-dimensional one.

We observe the formation of shocks in the solutions when the curve is not a graph anymore, i.e., a non-single-valued function. Once a shock happens, the characteristic's method is not valid anymore and the above expressions for $r(t)$ and $\rho(t)$ lack meaning. We also point out that the mass density, $r^{d-1} \rho$, is conserved up to the shock.

Cases 1 and 2 have initial profiles with supports of similar size, but case 2 presents shocks in a shorter time (both in 2-D and 3-D cases). This shows the dependence on the intensity of $\rho_{0}$; in case $2, \rho_{0}$ reaches 0.8 , while in case 1 , it is not greater than 0.35 . Case 1 and 2 illustrate the dependence on the value of $\rho_{0}$ for the time the shock appears. Both cases have initial profiles with supports of similar size, but case 2 presents shocks in a shorter time (both in 2-D and 3-D cases). In case $2, \rho_{0}$ reaches 0.8 , while in case 1 , it is not greater than 0.35 .

Finally, the presence of shocks in our examples gives the existence of shocks for the Hughes model in any dimension (for example, by looking at solutions that depend only on two or three dimensions in a higher-dimensional space). We are not aware of any proof of this feature in the literature.

\section{FLOW PROBLEM - STATIONARY CASE}

The flow problem is a natural problem in dimension one. It consists of people entering a domain from one side at a

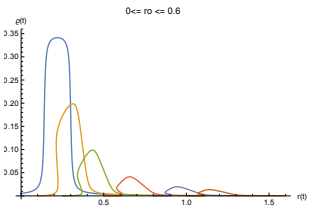

(a) Case 1: $\rho_{0}$ concentrated around 0.2 with support on $0 \leq r_{0} \leq 0.6$ and maximum value of 0.35 .

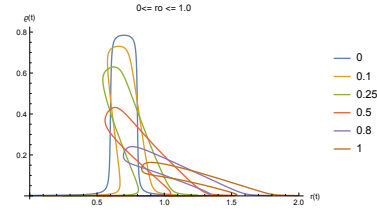

(b) Case 2: $\rho_{0}$ concentrated around 0.75 with support on $0.5<r_{0} \leq 1.0$ and maximum value of 0.8 .

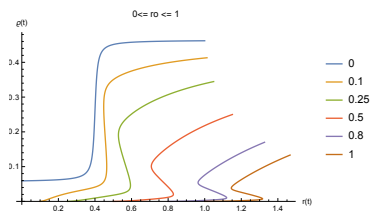

(c) Case 3: $\rho_{0}$ with support on $0 \leq r_{0} \leq 1.0$ and maximum value of 0.4 .

Fig. 2: Shocks along the time evolution of three given profiles in 3-D.

fixed rate and exiting through the other side. If the flow is large enough, the maximal density $\rho=1$ may be achieved or exceeded and the model may break.

In the one-dimensional flow problem in $[0,1]$, agents arrive at $x=0$ and are only allowed to leave through $x=1$. By computing $u_{x}$ in the Eikonal equation and substituting in the Fokker-Planck equation, we obtain

$$
\rho_{t}+\rho_{x}(1-2 \rho)=\varepsilon \rho_{x x} .
$$

The corresponding stationary equation is

$$
\varepsilon \rho_{x x}+2 \rho \rho_{x}-\rho_{x}=\frac{d}{d x}\left[\varepsilon \rho_{x}+\rho^{2}-\rho\right]=0 .
$$

We can then formulate the stationary flow problem as:

$$
\left\{\begin{array}{l}
\varepsilon \rho_{x}+\rho^{2}-\rho=j, \quad x \in[0,1], \\
\rho(1)=0,
\end{array}\right.
$$

where $j$ is a prescribed net current of agents entering $[0,1]$.

Our interest is to understand the behavior of the solutions of (15) as the current $j$ becomes large; that is, a large flow of agents. By solving numerically the ODE (15), for $\varepsilon=1$ and $j$ between 0 and 1.5, with increments of 0.1 , we observe the different solutions for $\rho$. For $j>1.2$, the density $\rho$ is larger than 1, see Figure 3a. This is remarkable because solutions of the reduced equation (15) are $C^{\infty}$, but in the original model the equations are singular when $\rho=1$.

In Figure 3b, we depict the effect of a small viscosity $(\varepsilon=$ 0.01 ) for a range of admissible currents (the ones avoiding $\rho>1$ ). The current for which the model reaches the maximal density 1 is called the critical current.

\section{A. Dependence on viscosity}

Here, we investigate the dependence of the solutions on the viscosity. In Figure 4a, we see that, for small viscosity, the model breaks down. However, large viscosity seems to 


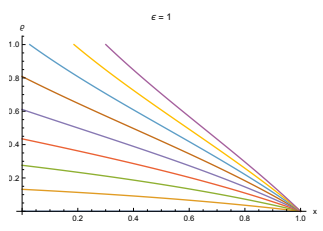

(a) $\varepsilon=1$ and $j$ from 0 to 1.5

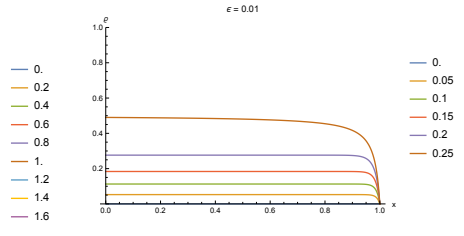

(b) $\varepsilon=0.01$ and $j$ from 0 to 0.25 .
Fig. 3: Onset of congestion.

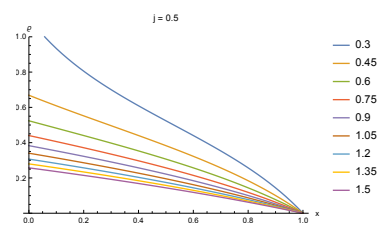

(a) Supercritical case

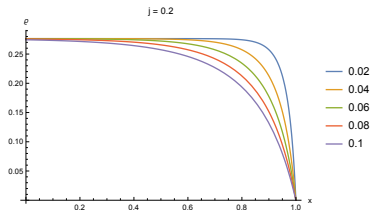

(b) Subcritical case
Fig. 4: Dependence of congestion on viscosity.

have a stabilizing effect. In Figure $4 a$, we used a current with a fixed value $j=0.5$ and viscosities from 0.3 to 1.5 with increments of 0.1 .

For $j=0.2$, the different solutions for the density $\rho$ show an upper bound when using different viscosities (from 0 to 0.1 with increments of 0.02 ), see Figure $4 \mathrm{~b}$. We remark that our choice of values for $j$ had only the goal of illustrating the different behaviors (15) can present.

To investigate the dependence of the critical current on the viscosity, we solve (15) with different viscosities and compute the critical current. The result is shown in Figure 5 .

\section{TREND TO EQUILIBRIUM}

We investigate the relation between the solution to the stationary problem (15) and the time-dependent one (14). The numerical solution to the stationary problem is calculated using an ODE solver. Now, the solution to the timedependent case is computed using the numerical approach further described in Section VI. As an example, we solve the problem with the following initial/boundary conditions, and $\varepsilon=0.05$ :

$\rho(0, t)=-0.2\left(1-e^{-10 t}\right), \rho(1, t)=0, \rho(x, 0)=x^{2}(1-x)^{2}$.

For large times, the stationary solution is an upper bound to the time-dependent one (the transient time in which this behavior fails is due to the initial condition). This behavior is depicted in Figure 6a. The time-dependent solution is depicted with the filled graph, while the plot of the stationary one uses a wire mesh.

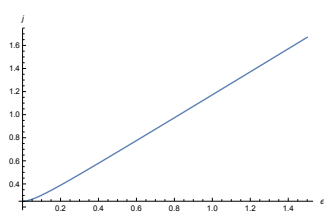

Fig. 5: Critical current $j$ as a function of viscosity.

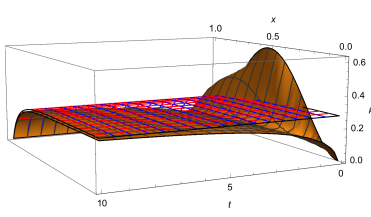

(a) Dominance

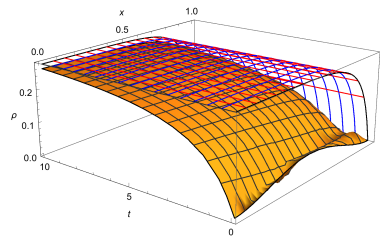

(b) Trend to the equilibrium
Fig. 6: Stationary and time-dependent solutions.

The second aspect we observe is the trend to equilibrium of the time-dependent solution in the subcritical case, Figure $6 \mathrm{~b}$, i.e. the asymptotic solution approaches the stationary solution for large times. We are not aware of any proof or theoretical result concerning this property.

\section{NUMERICAL APPROACH}

In contrast to the ODE numerical approach used in section IV, we present here a numerical PDE method to solve system (1). We first remark that the eikonal equation in (1) is a particular case of a Hamilton-Jacobi equation.

To construct a scheme that solves the Hughes model, we must use numerical methods that discretize $D u$ in a consistent way for both equations in (1). Our approach is the following: since the Fokker-Planck equation in (1) is the adjoint of the linearization of the nonlinear Hamilton-Jacobi operator

$$
-u_{t}+(1-\rho)^{2} \frac{|D u|^{2}}{2}-\varepsilon \Delta u,
$$

we can treat both equations, i.e., this new Hamilton-Jacobi equation (16) and the original Eikonal in (1) (seen as a Hamilton-Jacobi equation) via the same numerical method, for instance, using a monotone scheme.

In the examples of the next section, we use a space semidiscretization and treat the time variable as continuous (using a stiff solver for the numerical time evolution).

Let $h$ be the mesh size, $x_{n}$ the grid points and $u_{n}, \rho_{n}$ be the corresponding node values of $u$ and $\rho$. The nonlinearity in the Eikonal equation in (1) at a point $x_{n}$ is discretized through the monotone scheme

$$
\begin{aligned}
N_{n}(u) & \equiv \frac{\max \left\{u_{n}-u_{n-1}, 0\right\}^{2}}{h^{2}} \\
& +\frac{\max \left\{u_{n}-u_{n+1}, 0\right\}^{2}}{h^{2}}-\frac{1}{\left(1-\rho_{n}\right)^{2}} .
\end{aligned}
$$

The operator (16) is discretized as

$$
\begin{aligned}
\tilde{N}_{n}(u) \equiv & \left(1-\rho_{n}\right)^{2}\left[\frac{\max \left\{u_{n}-u_{n-1}, 0\right\}^{2}}{2 h^{2}}\right. \\
& \left.+\frac{\max \left\{u_{n}-u_{n+1}, 0\right\}^{2}}{2 h^{2}}\right]-\varepsilon \frac{u_{n+1}-2 u_{n}+u_{n-1}}{h^{2}} .
\end{aligned}
$$

The numerical scheme is then given as

$$
\left\{\begin{array}{l}
N(u)=0, \\
\rho_{t}+\left(D_{u} \tilde{N}(u)\right)^{T} \rho=0 .
\end{array}\right.
$$

Thanks to the adjoint structure, the second equation is discretized automatically: by taking the symbolic derivative 


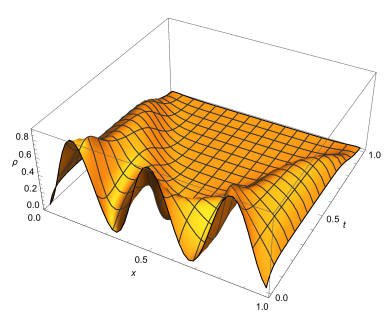

(a) Density $\rho$

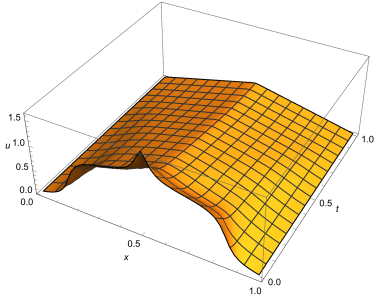

(b) Eikonal solution
Fig. 7: Numerical example 1.

of $\tilde{N}$ and due to the transpose, second equation in (17) corresponds to the discretization of the Fokker-Planck equation in (1). This approach is valid in arbitrary dimension and, by construction, conserves mass and positivity.

\section{NUMERICAL EXAMPLES}

Now, we use the numerical method of the previous section and present two examples for the 1-dimensional case. In the first one, agents/people/pedestrians can leave the domain from both sides. In the second example, we impose a flow of agents entering with reflecting boundary conditions on that side (preventing agents from leaving immediately) and Dirichlet conditions on the opposite side.

\section{A. Example 1}

For the first numerical example, we solve the Hughes model with a low viscosity $\varepsilon=0.01$, and the following initial/boundary conditions:

$$
\begin{cases}\rho(0, t)=0, & \rho(1, t)=0, \quad \rho(x, 0)=0.9 \sin ^{2}(3 \pi x), \\ u(0, t)=0, & u(1, t)=0 .\end{cases}
$$

These boundary conditions correspond to the exit problem. Agents have an initial distribution $\rho(x, 0)$ and seek to leave the interval $[0,1]$ by either $x=0$ or $x=1$. We plot the density $\rho$ and the solution $u$ in Figure 7 .

\section{B. Example 2}

The second example is the flow problem for the following initial/boundary conditions:

$$
\rho(1, t)=0, \rho(x, 0)=0.4 \sin ^{2}(3 \pi x), u(x, 1)=0 .
$$

In addition, at $x=0$, we impose for $\rho$ a flow-one condition:

$$
\rho(1-\rho)^{2} D u+\varepsilon \rho_{x}=1,
$$

and, at $x=0$, we impose reflecting boundary condition for $u$. Here, we use a higher viscosity, $\varepsilon=0.1$ and present our results in Figure 8.

\section{CONCLUSiOnS}

Here, we develop new a priori estimates for the Hughes model, which are an important step in understanding the wellposedness of the system for $\varepsilon>0$. Next, we use radial solutions to prove the existence of shocks in dimension greater than one. Consequently, the Hughes model without viscosity may fail to have smooth solutions. Then, we

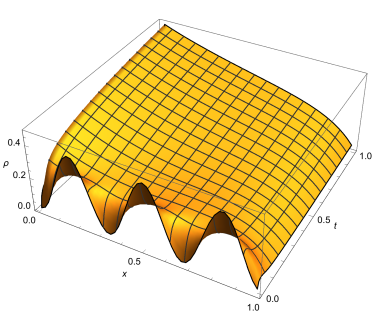

(a) Density $\rho$

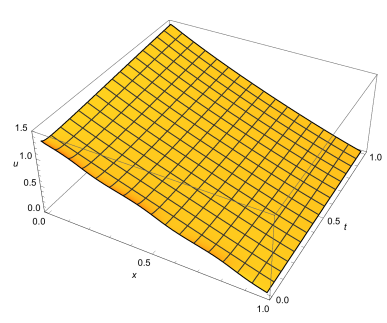

(b) Eikonal solution
Fig. 8: Numerical example 2.

uncover a new mechanism for the breakdown of classical solutions in the fixed current problem. Here, the critical density is reached without loss of regularity in $\rho$. Moreover, we examine the dependence of the critical current on the viscosity, and we present numerical evidence for the existence of a long-term limit. Finally, we describe a new method for the approximation of the Hughes model. Our method combines, in a novel way, monotone schemes for HamiltonJacobi equations with the adjoint structure of the FokkerPlanck equation, and applies to a wide range of related problems.

\section{REFERENCES}

[1] Debora Amadori and M. Di Francesco. The one-dimensional Hughes model for pedestrian flow: Riemann-type solutions. Acta Math. Sci. Ser. B Engl. Ed., 32(1):259-280, 2012.

[2] Debora Amadori, Paola Goatin, and Massimiliano D. Rosini. Existence results for Hughes' model for pedestrian flows. J. Math. Anal. Appl., 420(1):387-406, 2014.

[3] Martin Burger, Marco Di Francesco, Peter A. Markowich, and MarieTherese Wolfram. Mean field games with nonlinear mobilities in pedestrian dynamics. DCDS, 19(5):1311-1333, 2014.

[4] E. Carlini, A. Festa, F. J. Silva, and M.-T. Wolfram. A semi-lagrangian scheme for a modified version of the hughes model for pedestrian flow. Dynamic Games and Applications, 2016.

[5] Jose A. Carrillo, Stephan Martin, and Marie-Therese Wolfram. An improved version of the Hughes model for pedestrian flow. Math. Models Methods Appl. Sci., 26(4):671-697, 2016.

[6] M. Di Francesco, S. Fagioli, M. D. Rosini, and G. Russo. Deterministic particle approximation of the Hughes model in one space dimension. ArXiv e-prints, February 2016.

[7] Marco Di Francesco, Peter A. Markowich, Jan-Frederik Pietschmann, and Marie-Therese Wolfram. On the Hughes' model for pedestrian flow: the one-dimensional case. J. Differential Equations, 250(3):1334-1362, 2011.

[8] Nader El-Khatib, Paola Goatin, and Massimiliano D. Rosini. On entropy weak solutions of Hughes' model for pedestrian motion. $Z$. Angew. Math. Phys., 64(2):223-251, 2013.

[9] Paola Goatin and Matthias Mimault. The wave-front tracking algorithm for Hughes' model of pedestrian motion. SIAM J. Sci. Comput., 35(3):B606-B622, 2013.

[10] Diogo A. Gomes and João Saúde. Mean field games models-a brief survey. Dyn. Games Appl., 4(2):110-154, 2014.

[11] Roger L. Hughes. A continuum theory for the flow of pedestrians. Transportation Research Part B, pages 507-535, 2002.

[12] Filippo Santambrogio. A modest proposal for MFG with density constraints. Netw. Heterog. Media, 7(2):337-347, 2012. 